\title{
Integration features of control systems in multi- complex distributed production
}

\author{
Georgiy Makarov ${ }^{1 *}$, Leonid Myshlyaev ${ }^{1}$, Aleksandr Slamatin ${ }^{1}$, and Vladimir Vdovichenko ${ }^{2}$ \\ ${ }^{1}$ Research Center of Control Systems LLC, Novokuznetsk, 654005, Russia \\ ${ }^{2}$ Omron Electronics LLC, Moscow, 125040, Russia
}

\begin{abstract}
Different options of the structure of automated process control system (APCS) are considered using the example of the coal preparation plant with a single automation platform Omron Sysmac, as well as with heterogeneous devices connected by industrial networks Ethernet/IP at several levels with physical separation. The general APCS structure is proposed. It is suitable for the end-to-end control of objects with variable structure, built from various technological complexes of coal preparation plants.
\end{abstract}

\section{Introduction}

Modern production is characterized by the flow of technological processes in complexes that do not work as rigidly established sequential stages, but can also be combined to achieve the set goal. In the field of coal preparation, there are often parallel independent production branches, bypass paths for certain complexes, complexes for extraction and processing of specific classes of coal, controlled storage and mixing, etc. Such a controlled structure makes it possible to rebuild dynamically the production for various raw materials, the volumes and characteristics of the finished product specified by the plan, or withdrawal of any equipment for scheduled repair to obtain the highest efficiency. The control systems of these production facilities should be considered as systems with a variable structure [1] and take into account various kinds of arising feedforward and feedback [2].

The control of such systems should take into account the features noted above, predict the effects of decisions made at the level of production control, operators of technological processes, changes in the equipment operation or raw materials. For this purpose, specialized models are developed that form the basis of production digital twins. This allows us to speak of "end-to-end control", when changes in any part of the system make it possible to evaluate changes in others and make appropriate decisions. For example, when the general production plan changes, it is necessary to put into operation (or withdraw) certain production complexes and set the necessary agreed baseline levels of productivity for each technological unit in the chain. Such solutions are actively being developed in many industries, such as petrochemical, mechanical engineering, mining and processing. At the moment, the digital transformation is actively changing the metallurgy. For example, in [3], the main directions for application of "Industry 4.0" concept at Magnitogorsk Iron \&

* Corresponding author: mail@,nicsu.ru 
Steel Works PJSC are outlined, which allow creating a unified information basis for the implementation of a set of measures to optimize and increase the work efficiency of both separate units, workshops, production facilities and the enterprise as a whole.

\section{Integration based on a single platform}

To implement the principles of such end-to-end control, it is necessary to integrate all involved automation tools into a single system. Omron proposes to use the Sysmac platform to address these challenges (Fig. 1), which integrates motion control, programmable control, security, network control and visual control. EtherCAT is the fastest network of equipment that allows controllers, I/O baskets and devices to be connected in a single environment. IO-Link is a network communication protocol of point-to-point type for connecting sensors and actuators to a controller. Ethernet/IP is a common network protocol that allows visualization interfaces, servers and other controllers to be integrated.

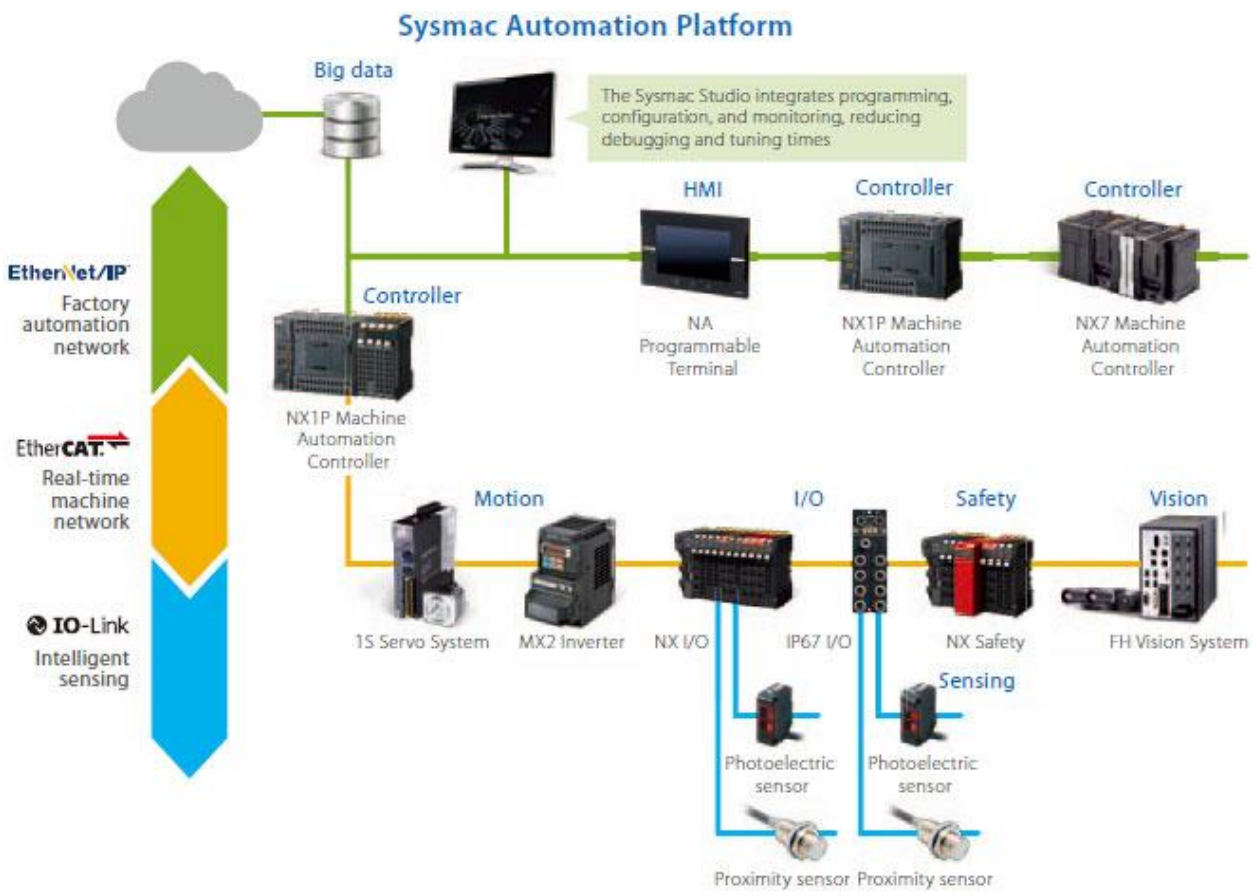

Fig. 1. Sysmac automation platform.

The use of Big data technologies and predictive diagnostics, implemented in intelligent modules built into the devices themselves [4], can significantly increase the level of transparency of processes and access to data. Such devices include both the universal machine controller with artificial intelligence Omron of NX and NY series and individual devices, for example, the K6CM monitoring devices used for assessing the condition of asynchronous motors, the K6PM-TH used for monitoring and analyzing the temperature status of panel devices to optimize energy efficiency and significantly reduce the risk of emergency shutdown. In addition to the built-in hardware for monitoring and diagnostics, it should also be noted that there is a need to develop built-in software modules and blocks that can be applied for operational diagnostics and assessment of pre-emergency states of technological equipment and supervisory instruments [5]. 
However, when designing such a large and complex facility as a coal preparation plant, it is often impossible to implement the entire system on the basis of one platform. Some devices are prepacked with technological equipment, some are purchased as a result of a technical error, etc. In this case, we have to talk about the integration of various devices, different manufacturers, on different protocols, separate local control systems with their own operating characteristics.

\section{Integration of various tools into a single system}

The structure of the main APCS must be able to connect such a variety of tools, but without compromising its own performance and safety. During the design of control system for the coal preparation plant "Mine No. 12" [6], the main control system was implemented on the Omron controllers of CJ2 series and the remote I/O of NX series. APCS networks have several levels and are physically separated using separate optical cores and controlled switches:

- FINS for communication of controllers with HMI and SCADA;

- Ethernet/IP for communication of controllers with each other and input/output;

- Modbus for communication with starting equipment, etc.

However, not all local systems were equipped with the ability to separate networks physically, and integration was required at both levels - operational dispatch monitoring and control and the level of programmable logic controllers. Controllers of some local systems also did not support FINS communications. The noted features require great flexibility and a wide range of capabilities from the designed APCS.

Division of production into complexes and lines, when it is necessary to make a decision on the distributed implementation of information and control functions, i.e. distributed I/O or control has a significant impact on the development of the APCS structure [7]. Large complexes, such as a drying-furnace section or a flotation section, may have a small number of adjacent units and signals with the rest of the technological complexes. At the same time, they can work separately "modifying" the available volumes of products, regardless of the work of the rest facilities. In this case, it is advisable to talk about a separate control controller and a control system for them.

The advantages of a separate control system increase the system reliability, makes it possible to carry out any work in the complexes without interrupting the work of the rest, to reduce the amount of information continuously requested by the dispatching system from the controller.

In its turn, if the complexes have a large number of adjacent units distributed over the distribution points, buildings (or parts of a building), and the start-up and blocking of the technological chain is not carried out sequentially in each complex, but in accordance with the complex schemes and dependencies, it is more rational to combine them with a single control system using a controller with remote $\mathrm{I} / \mathrm{O}$.

This approach has the following advantages:

1) improves the system reliability;

2) reduces the cost of the system;

3) processor module is not bound to managed objects, data about their condition can be transmitted over long distances.

4) implementation of the control algorithm for the entire industrial facility in a single processor module leads to a decrease in the factory downtime during its operation.

The system of Omron NX remote I/O, built on the EtherCAT network, has a high data exchange rate and is applicable for processes when a prompt response is required to the smallest and fastest changes in their parameters, and the slightest changes in accuracy lead to rejects. However, the disadvantage of this system is the impossibility to take a separate 
node (I/O basket) out of operation without stopping the operation of all other nodes. But for an operating coal preparation plant such a need often arises, since the equipment can fail and requires prompt replacement and reassembly. Often new devices are added in the conditions of functioning production. This requires flexibility of the control system and the facility proving the option to quickly switch and bypass routes.

In order to ensure the independent operation of all nodes - local systems, I/O baskets, it is necessary to integrate them through networks, allowing both ring structures and a "star" structure to be implemented. In the Omron NX product line, it is possible to integrate both I/O baskets and controllers over an industrial Ethernet/IP network. However, in this case, the problem of access control and physical division of networks into several levels arises. A possible structure configuration of the APCS control part at the coal preparation plant for such a case is shown in Fig. 2.

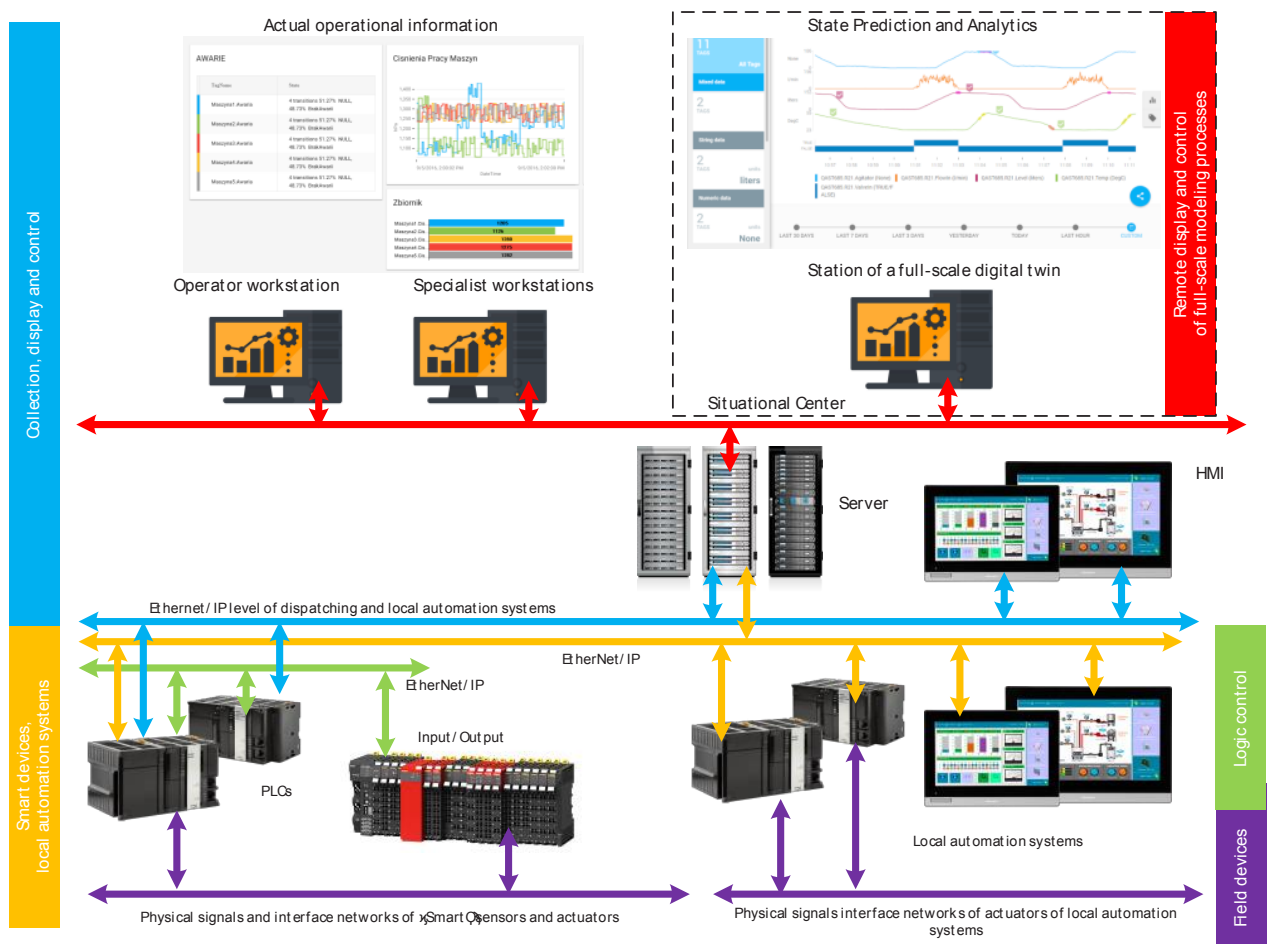

Fig. 2. Example of the structure of APCS control part.

\section{Conclusion}

The proposed structure makes it possible to achieve modularity, expandability of the system and organize optimally the functions of distributed $\mathrm{I} / \mathrm{O}$ and control. Such a technical structure should underlie the modern coal preparation plant APCS, divided into complexes and lines which characterize production as an object with a variable structure. Objects with variable structure require, in turn, special control systems and regulating loops designed not only for coordinate-parametric control, but also for controlling the structure of the object and the system itself [2]. When designing and configuring these systems, it is also necessary to take into account the effects arising from various types of control connections. This is relevant both for local control loops and for the entire object as a whole. 
The "manually" started continuous handling systems are limited to the sequence of actions performed by one operator, or "on-site" machine operators. At the same time, the automatic launch control system can put into operation unrelated complexes and units in parallel, take into account the necessary launch sequences and the values of technological parameters, and independently proceed to the next step immediately after the disappearance of technological interlocks. This makes it possible to significantly reduce the start-up time of the processing plant. For example, if a plant operator needs 40 to 60 minutes to start from scratch, then the automatic launch control system allows launching in 15 minutes, which significantly changes the dynamics of the factory complexes as control objects.

For individual units and circuits, the problem of creating and configuring control systems can be solved in various ways, each of which has its own area of effective application. The typification of technological units from the control point of view [8] makes it possible to implement both typical automatic control systems and logical control systems. Omron controllers starting with the CJ2 series support not only generic function blocks, but also the organization of $\mathrm{I} / \mathrm{O}$ and state variables in the form of data structures. An example of such typing based on the Omron CJ2H controller is patented as a computer software [9]. To create and configure control systems for more complex circuits, or processes that do not have valid examples, experimental physical models or semi-industrial installations are created. Adequate transfer of the results of control systems research on such models is possible only when using the methods of the theory of control systems similarity [10].

The use of the approaches and tools noted in the article allows both new control automation systems and modernize existing ones to be effectively created and implemented, gradually introducing elements of end-to-end control, full-scale digital twins and Industry 4.0 .

\section{References}

1. S.V. Emelyanov, Automatic control systems with variable structure (Moscow, Nauka, 1967)

2. S.V. Emelyanov, S.K. Korovin, New types of feedback: Control under uncertainty (Moscow, Nauka, Fizmatlit, 1997)

3. N.A. Spirin, V.V. Lavrov, et al, Concept of Industry 4.0. Scientific problems in creating intelligent control systems for technological processes in pyrometallurgy, in Proceedings of the XII Conference on Automation Systems in Education, Science and Production, AS'2019, Novokuznetsk (2019)

4. S.P. Romanovsky, V.A. Akhmarov, Practical aspects of the implementation of elements of digital machines in the framework of the concept "Industry 4.0", in Proceedings of the XII Conference on Automation Systems in Education, Science and Production, AS'2019, Novokuznetsk (2019)

5. M.V. Lyakhovets, G.V. Makarov, et al, Industrial ACS and controllers, 3, 16-24 (2020)

6. M.V. Shipunov, V.V. Grachev, et al, Automation control system for the concentration plant LLC "Mine No. 12", in Proceedings of the XII Conference on Automation Systems in Education, Science and Production, AS'2019, Novokuznetsk (2019)

7. A.S. Salamatin, G.V. Makarov, M.V. Raskin, Features of distributed management of industrial facilities, in Proceedings of the Conference of Students, Postgraduates and Young, Novokuznetsk (2019)

8. A.S. Salamatin, G.V. Makarov, et al, Typical solutions for the automation of technological facilities on the example of coal preparation plants, in Proceedings of 
the Conference on Science-intensive technologies for the development and use of mineral resources, Novokuznetsk, (2018)

9. L.P. Myshlyaev, G.V. Makarov, et al, Patented software: Typical functional block for controlling valves, valves with discrete control (2021)

10. V.F. Evtushenko, L.P. Myshlyaev, et al, Research of control systems using physical models, in Proceedings of the X Conference on Automation Systems in Education, Science and Production, AS'2015, Novokuznetsk (2015) 